\title{
Dolor retroesternal y fiebre con síntomas catarrales; un caso de mediastinitis por Haemophilus influenzae
}

\author{
Marta Torres-Arrese, Carolina Hernández-Durán, Adrián Moya-Barrientos, Lorena Castro-Arias \\ Servicio de Urgencias. Hospital Universitario Doce de Octubre. Madrid. España
}

Recibido:07/11/2018

Aceptado: 27/01/2019

En línea: 30/04/2019

Citar como: Torres-Arrese M, Hernández-Durán C, Moya-Barrientos A, Castro-Arias L. Dolor retroesternal y fiebre con síntomas catarrales; un caso de mediastinitis por Haemophilus influenzae. Rev Esp Casos Clin Med Intern (RECCMI). 2019 (Abr); 4(1): 3-5. doi: 10.32818/reccmi.a4n1a2.

Autor para correspondencia: Marta Torres-Arrese. martatorresarrese@gmail.com

\section{Palabras clave \\ $\triangleright$ Mediastinitis \\ $\triangleright$ Haemophilus influenzae \\ $\triangleright$ Pericarditis}

\begin{abstract}
Resumen
El caso que acontece pretende enfatizar en una patología potencialmente mortal. La demora en el diagnóstico de esta entidad influye tanto en el manejo como en el pronóstico. Un varón de 38 años sin antecedentes acudió al Servicio de Urgencias por fiebre, síntomas catarrales altos y dolor torácico retroesternal. Se encontraba estable, pero febril, y la analítica reveló elevación de reactantes de fase aguda. Se solicitó TAC, bajo la sospecha de mediastinitis, que confirmó el diagnóstico. Se inició antibioterapia y se tomó una actitud expectante. La precocidad del diagnóstico permitió un tratamiento conservador.

Abstract
The case that happens intends to emphasize a potentially fatal pathology. The delay in the diagnosis of this
entity potentially influences both the management and the prognosis. A 38-year-old male with no history
goes to the Emergency Department for fever, high catarrhal symptoms and retrosternal chest pain. He was
stable but febrile and the analytical revealed elevation of acute phase reactants. A CT scan was requested un-
der suspicion of mediastinitis that confirmed the diagnosis. Antibiotic therapy was initiated and an expectant
attitude was taken. The precocity of the diagnosis allowed a conservative treatment.
\end{abstract}

\section{Puntos destacados}

$\triangleright$ La mediastinitis aguda descendente es una entidad rara pero cuya detección a tiempo cambia el manejo y el pronóstico.

$\triangleright$ En nuestro caso, la sospecha precoz permitió el abordaje con antibioterapia y evitó una cirugía de elevada morbilidad y mortalidad.

$\triangleright$ La coexistencia con pericarditis por irritación por contigüidad, aunque descrita, es extremadamente infrecuente.

\section{Introducción}

La mediastinitis es una rara afección con morbilidad y mortalidad significativas cuya demora diagnóstica cambia el tratamiento y empeora drásticamente el pronóstico. En nuestro medio, la mayoría de las veces la observamos en contexto posquirúrgico (cirugía cardíaca) o como consecuencia de intervenciones como mediastinoscopias o gastroscopias con perforación esofágica secundaria. En los casos en los que no existe un contacto con el medio sanitario previamente, casi la mitad de las veces tiene un origen odontogénico. Otros orígenes son el faríngeo y el amigdalino. Respecto a la etiología, suelen ser polimicrobianas, y los microorganismos más frecuentemente aislados son los estreptococos del grupo Viridans. Estafilococos y gramnegativos también pueden estar presentes ${ }^{1-5}$. La infección del mediastino se inicia como una celulitis que con frecuencia degenera en necrosis que se extiende con poca o nula presencia de pus. Durante esta fase temprana, el tratamiento comporta antibioterapia y una cirugía demasiado precoz puede empeorar la situación al romper las defensas naturales. Una vez superada esta etapa, es preciso un desbridamiento quirúrgico adyuvante; y la mortalidad y la morbilidad aumentan considerablemente ${ }^{6}$.

\section{Historia clínica y exploración física}

Varón de 32 años, natural de Colombia (último viaje a su país natal hace 6 meses), sin antecedentes familiares ni personales de interés, que comenzó con un cuadro de odinofagia y fiebre hace 2 días junto con deterioro del estado general y paulatinamente dolor retroesternal que se incrementaba al deglutir, así como tos seca en las últimas horas. Negaba haberse atragantado, aunque había comido pescado previamente al inicio del cuadro.

A la exploración, el paciente se encontraba hemodinámicamente estable, con tensión arterial de 130/60 mmHg, frecuencia cardíaca de 110 latidos 
Torres-Arrese M, Hernández-Durán C, Moya-Barrientos A, Castro-Arias L.

Dolor retroesternal y fiebre con síntomas catarrales; un caso de mediastinitis por Haemophilus influenzae

por minuto y saturación de oxígeno del 97\% respirando aire ambiente. La temperatura era de $40{ }^{\circ} \mathrm{C}$. El paciente presentaba mal aspecto general, con sudoración profusa, aunque se encontraba eupneico y bien perfundido. La exploración faríngea reveló una faringe eritematosa brillante, con amíg dalas aumentadas de tamaño sin placas y úvula desviada a la derecha sin evidencia de abscesos. Presentaba adenopatías múltiples de alrededor de $1,5 \mathrm{~cm}$, de dominio izquierdo, lisas y rodaderas, no adheridas y levemente dolorosas a la palpación. El resto de la exploración era normal. Se solicitó valoración por Otorrinolaringología, que le diagnosticó rinosinusitis y faringitis aguda sin aparentes complicaciones.

\section{Pruebas complementarias}

La analítica mostraba aumento de reactantes de fase aguda (17.000 leucocitos/ $\mu$ l, con predominio de neutrófilos), proteína c reactiva (PCR) $28 \mathrm{mg} / \mathrm{dl}$, procalcitonina $6,6 \mathrm{ng} / \mathrm{ml}$, y coagulopatía (INR 1,6, TTPA 35 s). Se realizó electrocardiograma (ECG) que evidenció un ritmo sinusal sin alteraciones en la conducción ni en la repolarización a 100 lpm.

Dada la sospecha clínica de mediastinitis aguda, se solicitaron hemocultivos, serología de VIH y tomografía axial computarizada (TAC) torácica y cervical que mostró estriación de la grasa en el mediastino, tanto a nivel cervical como torácico, sin evidencia de colecciones (Figura 1) ${ }^{3}$, como sería esperable en un estadio más avanzado de origen otorrinolaringológico.

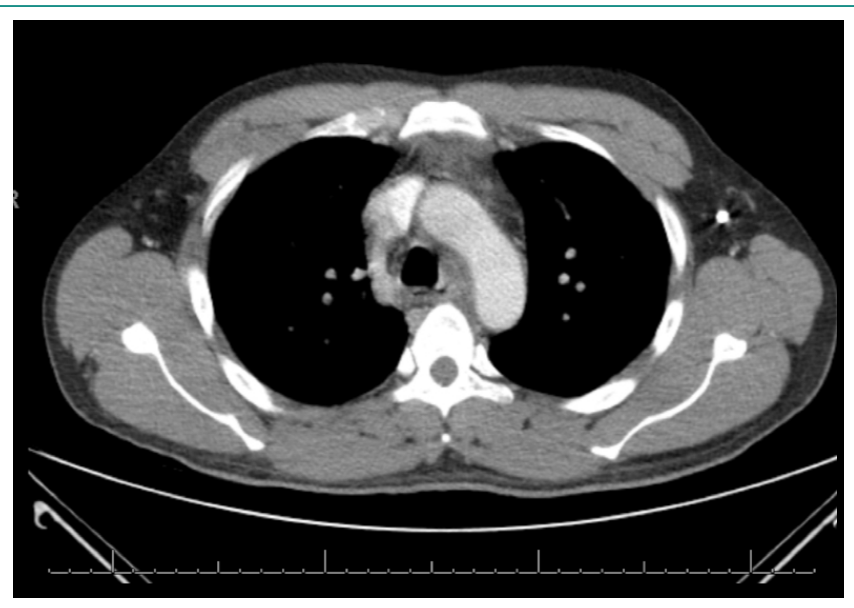

Figura 1. TAC: estriación de la grasa del mediastino

\section{Evolución}

Se inició antibioterapia de amplio espectro (piperacilina-tazobactam y amikacina), pese a lo cual, en las siguientes 12 horas y persistiendo el mismo tipo de dolor torácico, el paciente sufrió cuadro hipotensivo y requirió sueroterapia y fármacos vasoactivos. En este momento, se realizó nuevo ECG que evidenció cambios electrocardiográficos con T picudas y descenso del segmento PR, compatible con pericarditis aguda en probable relación con afectación por continuidad. El ecocardiograma urgente no visualizó derrame pericárdico. Dada la situación, el paciente ingresó en la unidad de cuidados intensivos (UCI) y se asociaron ibuprofeno y colchicina.

La radiografía de control ya mostraba ensanchamiento mediastínico incipiente. En los hemocultivos se aisló Haemophilus influenzae sensible a cefalosporinas, por lo que se decidió desescalar a ceftriaxona. La serología de VIH resultó negativa y, durante su estancia en $\mathrm{UCl}$, se realizó una gastroscopia que no mostró hallazgos.

A las 24 horas, se procedió a realizar TAC de control que manifestó un empeoramiento de la estriación de la grasa y derrame pleural de nueva aparición. Sin embargo, dada la situación de estabilidad hemodinámica y mejoría clínica, se procedió a trasladar al paciente a planta de hospitalización, donde el dolor torácico remitió. La evolución fue favorable y, cumpliendo 4 semanas de tratamiento antibiótico, el paciente fue dado de alta.

\section{Diagnóstico}

Mediastinitis aguda precoz de origen otorrinolaringológico por Haemophilus influenzae con pericarditis secundaria.

\section{Discusión}

Nuestro caso se trataba, por tanto, de una mediastinitis aguda por Haemophilus influenzae de etiología descendente, sin evidencia de absceso a nivel otorrinolaringológico en las pruebas de imagen. Como problemas secundarios, el paciente presentaba pericarditis aguda y derrame pleural leve por continuidad.

Como se pone de manifiesto en la revisión sobre la fascitis necrotizante cervical de Gunaratne et al., la presencia de fiebre y dolor retroesternal es una asociación que nos debe hacer pensar en mediastinitis ${ }^{1}$. Los antecedentes como una infección odontogénica, faríngea o amigdalar, un atragantamiento o un procedimiento sobre la zona (como gastroscopias o mediastinoscopias) nos tienen que poner alerta. El método diagnóstico de elección es la TAC.

En nuestro caso, el paciente presentaba un síndrome catarral previo sin aparentes complicaciones a nivel otorrinolaringológico, e ingesta de pescado en los días previos. La TAC únicamente evidenció estriación de la grasa, hallazgo sugestivo de mediastinitis inicial. En casos más avanzados, es frecuente la objetivación de colecciones o abscesos. El diagnóstico temprano en esta entidad cambia radicalmente el pronóstico y el manejo, sin embargo hay muy pocas referencias bibliográficas sobre el manejo conservador de esta entidad ya que, en la mayoría de ocasiones, se llega tarde al diagnóstico7,8. En esta ocasión, fue la sospecha inicial alta lo que permitió un manejo con antibioterapia. Respecto al tratamiento médico, dado que suelen ser polimicrobianas, se deben emplear antibióticos de amplio espectro y a dosis plenas². Regímenes recomendados son piperacilinatazobactam o clindamicina junto con metronidazol. El tratamiento inicial se realizó con piperacilina-tazobactam y amikacina, desescalando a ceftriaxona tras el aislamiento y el antibiograma. Es preciso comentar que la coexistencia con pericarditis y derrame pleural, aunque excepcional, está descrita y se debe plantear el drenaje. La evolución fue favorable y el paciente fue dado de alta.

\section{Bibliografía}

1. Gunaratne DA, Tseros EA, Hasan Z, Kudpaje AS, Suruliraj A, Smith MC, Riffat F, Palme CE. Cervical necrotizing fasciitis: systematic review and analysis of 1235 reported cases from the literature. Head Neck. 2018 Sep; 40(9): 20942102. doi: 10.1002/hed.25184.

2. Chow AW. Deep neck space infections. Uptodate.com [Internet]. Actualizado: Oct 11, 2017. Revisado: Oct 15, 2018. Disponible en: https:// www-uptodate-com.m-hdoct.a17.csinet.es/contents/deep-neck-spaceinfections? search=mediastinitis\%20aguda\&source=search_result\&selecte $\mathrm{dTitle}=2 \sim 150 \&$ usage_type $=$ default\&display_rank=2\#H34 
Torres-Arrese M, Hernández-Durán C, Moya-Barrientos A, Castro-Arias L. Dolor retroesternal y fiebre con síntomas catarrales; un caso de mediastinitis por Haemophilus influenzae

3. Brook I, Frazier EH. Microbiology of mediastinitis. Arch Intern Med. 1996 Feb 12; 156(3): 333-336.

4. Hurley MC, Heran MK. Imaging studies for head and neck infections. Infect Dis Clin North Am. 2007 Jun; 21(2): 305-353, v-vi.

5. Todd JK. Bacteriology and clinical relevance of nasopharyngeal and oropharyngeal cultures. Pediatr Infect Dis. 1984 Mar-Apr; 3(2): 159-163.

6. Rega AJ, Aziz SR, Ziccardi VB. Microbiology and antibiotic sensitivities of head and neck space infections of odontogenic origin. J Oral Maxillofac Surg. 2006 Sep; 64(9): 1377-1380.
7. Reynolds SC, Chow AW. Severe soft tissue infections of the head and neck: a primer for critical care physicians. Lung. 2009 Sep-Oct; 187(5): 271-279. doi: 10.1007/s00408-009-9153-7.

8. Chen KC, Chen JS, Kuo SW, Huang PM, Hsu HH, Lee JM, Lee YC. Descending necrotizing mediastinitis: a 10-year surgical experience in a single institution. J Thorac Cardiovasc Surg. 2008 Jul; 136(1): 191-198. doi: 10.1016/j. jtcvs.2008.01.009 